\title{
ORIGINAL
}

\section{DISTRIBUCIÓN TEMPORAL DE LAS DENUNCIAS Y MUERTES POR VIOLENCIA DE GÉNERO EN ESPAÑA EN EL PERÍODO 1998-2006}

\section{Carmen Vives-Cases (1, 2), Jordi Torrubiano-Domínguez (1) y Carlos Álvarez-Dardet (1, 2, 3)}

(1) Área de Medicina Preventiva y Salud Pública. Universidad de Alicante. Alicante. España.

(2) Ciber de Epidemiología y Salud Pública (CIBERESP). España.

(3) Observatorio de Políticas Públicas y Salud (OPPS).

\section{RESUMEN}

Fundamento: la violencia del compañero íntimo contra las mujeres (VCI) ha recibido especial dedicación de la agenda política española. A pesar de la importancia de las intervenciones desarrolladas, la evidencia empírica sobre su efectividad es todavía escasa. El objetivo de este estudio es explorar la distribución temporal de las denuncias y muertes por VCI e identificar posibles cambios de tendencia en las muertes y denuncias por VCI a partir de la Ley española contra la violencia de género de diciembre de 2004.

Métodos: estudio descriptivo de denuncias y muertes por VCI (1998-2006) basado en las estadísticas del Instituto de la Mujer y la Federación de Mujeres Separadas y Divorciadas. Cálculos: Tasas de mortalidad ajustadas por edad y tasa de denuncias por años y periodos en torno a la ley (1998-2004 vs. 20052006); Regresión de Poisson; e, Índices epidémicos mensual -razón entre casos mensuales y mediana de casos de los meses correspondientes al quinquenio anterior al mes para el que se calcula el índice- y subyacente -media anual de las puntuaciones del índice epidémico mensual- de denuncias y muertes.

Resultados: Desde 2005, las puntuaciones de los índices epidémicos muestran una tendencia decreciente. Sin embargo, la posibilidad de denunciar VCI entre 2005 y 2006 es 1,6 veces superior que entre 1998 y 2004 . El riesgo de morir por esta causa no muestra cambios estadísticamente significativos.

Conclusión: con el paso del tiempo se ha incrementado la posibilidad de denunciar VCI en España. Sin embargo, el riesgo de morir se mantiene. Aunque todavía es pronto para la evaluación del impacto de la ley en esta epidemia, puede decirse que su eficacia para la reducción de la mortalidad por $\mathrm{VCI}$ parece limitada.

Palabras clave. Mujeres maltratadas. Violencia doméstica. Mortalidad. Incidencia.

Correspondencia:

Carmen Vives Cases.

Dpto. Salud Pública. Edificio de Ciencias Sociales.

Campus Sant Vicent del Raspeig

Universidad de Alicante

Ap. 99. 03080 Alicante

Correo electrónico: carmen.vives@ua.es
ABSTRACT

\section{Temporary Distribution of Reports and Murders because of Partner Violence during the Period 1998-2006, Spain}

Background: Intimate Partner Violence (IPV) has received special attention in the spanish political agenda. Despite the importance of developed interventions, empirical evidence about their effectiveness is scarce. The aim of this study is to explore the temporary distribution of Intimate Partner Violence (IPV) reports and murders and to identify possible changes in the risk of dying and reporting by IPV from the Spanish law against gender violence of December of 2004.

Methods: We performed a descriptive study based on stadistics of The Woman's Institute and The Divorced and Separated Women Federation. Calculations: IPV mortality and reports rates by years and periods around the law (1998-2004 vs. 2005-2006); Poisson Regression; and, Epidemic index by months -Ratio between the actual number of IPV murders and reports in a given month and the median number of cases in the same month in the five preceding years- and underlying epidemic index -annual average of the scores of the epidemic index of reports and murders.

Results: The epidemic index trends permit us to observe that the problem has decreased since 2005 . Nevertheless, the posibility of reporting IPV between 2005 and 2006 is 1.6 times upper that between 1998 and 2004. The risk of dying by this cause does not show statistically significant changes.

Conclusion: The posibility of reporting IPV has been increased in Spain throughout the time. However, the risk of dying stays. Although still it is soon for the evaluation of the impact of the law in this epidemic, we could say that its effectiveness for the reduction of IPV mortality seems limited.

Key words: Battered women. Domestic violence. Mortality. Incidence. 


\section{INTRODUCCIÓN}

La violencia del compañero íntimo (VCI) es la más común en la vida de las mujeres, mucho más que las agresiones o violaciones perpetradas por extraños o conocidos $^{1}$. Se ha estimado que entre $13 \%$ y el $61 \%$ de las mujeres ha sufrido algún tipo de maltrato físico por parte de su pareja en algún momento de su vida ${ }^{2}$. Además, en la literatura científica, se sugiere la existencia de un "efecto acumulativo" de las diversas tipologías de maltrato, de manera que el impacto en la salud es todavía mayor en mujeres que experimentan conjuntamente abusos físicos, sexuales y psicológicos ${ }^{3,4}$.

Sólo teniendo en cuenta la violencia física contra las mujeres, se ha cuantificado un impacto económico anual en 5,8 billones de dólares en Estados Unidos ${ }^{5}$. Se trata de una cifra parecida a la del Ministerio de Industria y Comercio de Gran Bretaña, incluyendo no sólo los gastos económicos directos generados en servicios de salud, sino también aquellos relacionados con el sistema judicial y los servicios sociales. Si bien en este último caso se trata de costes directos que reúnen en total 3,1 billones de libras en 2004, aquellos generados indirectamente por la pérdida de productividad potencial casi sextuplican la cifra alcanzando los 17 billones de libras anuales ${ }^{6}$.

La gravedad del problema para la salud, el bienestar y los derechos de las mujeres y su impacto económico y social explican por sí mismos su progresiva incorporación como tema de la agenda de las instituciones políticas ${ }^{7}$. En España, en las últimas tres décadas, y sobre todo desde mediados de los noventa, se ha observado un progresivo proceso de construcción de políticas sobre el problema. En el año 1998 se definió el denominado I Plan de Acción contra la Violencia Doméstica (1998-2000) ${ }^{8}$, seguido del II Plan Integral contra la Vio- lencia Doméstica (2001-2004) ${ }^{9}$. Finalmente, a finales de 2004, se aprobó la Ley Orgánica 1/2004 de Medidas de Protección Integral contra la Violencia de Género ${ }^{10}$. Esta nueva legislación contiene las singularidades de hacer referencia expresa a la violencia de género o violencia contra las mujeres y de plantear medidas que comprometen a diferentes instituciones públicas (administrativa, penal, laboral, educativa, sanidad) ${ }^{11}$. Al igual que ocurre con la legislación sobre el tema existente en otros países, todas estas medidas se han caracterizado por poner de manifiesto el carácter delictivo específico de la violencia de género y, por tanto, apoyar la interposición de denuncias por parte de las víctimas $^{12}$.

Una de las características diferenciales de la Ley española contra la violencia de género es precisamente su denominación, violencia de género, que sólo comparte con la ley de Suecia $^{13}$. La interpretación jurídica de este concepto en la ley española es el reconocimiento de las mujeres como ciudadanas equiparadas a los "ciudadanos" y el énfasis en el deber del Estado de garantizar a las mujeres el pleno ejercicio de los derechos fundamentales. Además, en la ley española se hace explícito este reconocimiento planteando derechos laborales acordes a las circunstancias de aquellas trabajadoras afectadas por la violencia de género, derechos económicos con el fin de facilitar su integración social y el derecho a la información para asegurar a las víctimas de violencia de género un acceso rápido, transparente y eficaz a los servicios públicos ${ }^{10}$. Podría decirse que todas estas medidas también incorporan novedades en términos de reducción del riesgo de recurrencias y agravamiento del problema, que a su vez complementan otras estrategias de prevención primaria también contempladas en las leyes de otros países ${ }^{14}$.

A pesar de la importancia de las intervenciones desarrolladas en torno a este 
problema, la evidencia empírica existente sobre la efectividad de las leyes y políticas en torno a la VCI es todavía escasa ${ }^{15,16}$. Destacan, en este sentido, los estudios sobre el impacto de las órdenes de alejamiento y restricción de posesión de armas en la prevención de femicidios perpetrados por las parejas ${ }^{17-21}$. En España, hay estudios posteriores a la aprobación de la ley contra la violencia de género sobre las estrategias desarrolladas por las mujeres afectadas $^{22,23}$. Sin embargo, al igual que ocurre en el contexto internacional, la efectividad de las intervenciones programadas en los términos de reducción del riesgo de recurrencia del problema y de mortalidad por esta causa no ha sido suficientemente evaluada.

Aunque se cuenta con un informe provisional realizado por la Delegación Especial del Gobierno español contra la Violencia de Género sobre la puesta en marcha de las medidas planteadas en la ley $^{24}$, se desconoce el impacto de estas medidas sobre la evolución del problema. A pesar de que resulta difícil la realización de una evaluación de la ley por la novedad de esta iniciativa, la importancia de la nueva legislación merece una primera aproximación a sus efectos en el perfil epidemiológico de la VCI en España.

El objetivo de este estudio es explorar la frecuencia de las denuncias y muertes por VCI a lo largo del tiempo e identificar posibles cambios de tendencias a raíz de Ley Orgánica 1/2004 de Medidas de Protección Integral contra la Violencia de Género $^{10}$.

\section{MATERIAL Y MÉTODOS}

Se realizó un estudio epidemiológico descriptivo de las denuncias y muertes de mujeres por VCI ocurridas en España entre 1998 y 2006.
Para los datos de muertes por VCI se consultó la base de datos de la Federación de Mujeres Separadas y Divorciadas, que recoge y publica mensualmente en su página $w_{e} b^{25}$ las muertes de mujeres víctimas de violencia de género que son cubiertas por la prensa española. Se seleccionaron aquellos casos relacionados con la definición de Violencia del Compañero Íntimo contra las mujeres (VCI), referida a "cualquier comportamiento dentro de una relación íntima presente o anterior que causa daño físico, psíquico o sexual" de hombres contra mujeres ${ }^{1}$.

Las denuncias se tomaron de la página web del Instituto de la Mujer ${ }^{26}$. Los datos recogidos desde 1998 a 2005 son de elaboración propia del Instituto de la Mujer a partir de noticias de prensa y de las estadísticas del Ministerio del Interior. A partir del año 2006, los datos son recogidos por la Delegación Especial del Gobierno contra la Violencia sobre la Mujer. En las estadísticas del Instituto de la Mujer sólo se incluyen aquellos casos en los que el agresor es el cónyuge, ex cónyuge, compañero sentimental, ex compañero sentimental, novio o ex novio.

Aunque el Ministerio del Interior cuenta con datos de muertes por VCI, la Federación de Mujeres Separadas y Divorciadas ofrece una descripción más detallada de estos sucesos que permiten calcular indicadores más precisos. En concreto, en la página Web de la Federación de Mujeres Separadas y Divorciadas se facilita información sobre la fecha de ocurrencia del suceso, el periódico que recoge la noticia, nombre de la víctima, edad de la mujer asesinada, ciudad en la que tuvo lugar el suceso, parentesco de la víctima con el agresor y una breve descripción de los hechos ocurridos. Tal y como se ha puesto de manifiesto en estudios anterio$\operatorname{res}^{27,28}$, las estadísticas de muertes por VCI de la Federación de Mujeres Separa- 
das y Divorciadas coinciden con las del Ministerio del Interior en el $89 \%$ de los casos registrados anualmente para el periodo 1999-2006.

En total, se recogieron 360.830 denuncias por VCI de las estadísticas del Ministerio del Interior y 484 muertes de mujeres a manos de sus parejas o análogos de las de la Federación de Mujeres Separadas y Divorciadas.

Se calcularon tasas de mortalidad y denuncias por VCI por años (desde 1998 hasta 2006) y entre los periodos 1998-2004 vs. 2005-2006 en torno a la aprobación de la Ley Orgánica 1/2004 de Protección Integral contra la Violencia de Género en diciembre de $2004^{10}$.

En el caso de las denuncias se calcularon tasas brutas porque las estadísticas del Ministerio del Interior no ofrecen los datos desagregados por edad. Para el cálculo de las tasas brutas de denuncias por VCI se consideró en el numerador el total de denuncias producidas en el periodo estudiado (años y periodos en torno a la ley contra la violencia de género) y en el denominador la población de mujeres españolas de 15 años o más según el padrón municipal español ${ }^{29}$. En el caso de las tasas anuales, se tomó como población estándar el número de mujeres españolas de 15 años o más en 2002. En la tasa del periodo comprendido entre 1998 y 2004 se tomó como población de referencia la del año 2001. Por último, en el caso de la tasa del periodo 2005-2006 la población estándar fue la del año 2006.

Por su parte, en el caso de las muertes por VCI se calcularon tasas ajustadas por edad para cada uno de los periodos de estudio (anuales y en torno a la ley contra la violencia de género) por el método directo $^{30}$. En el denominador de las tasas ajustadas (personas-tiempo) las poblaciones de referencia fueron las mismas utilizadas en el cálculo de las tasas de denuncias. Se introdujo la suma de las poblaciones de referencia multiplicadas por los años comprendidos en cada periodo de estudio. Para el numerador, se calculó la suma de las muertes esperadas por VCI para cada edad en cada uno de los periodos de estudio.

Además, se utilizó el modelo de Poisson Para testar hipótesis sobre variación de la tendencia temporal tanto de muertes como de denuncias después de la promulgación de la ley. En este caso también se hizo la agrupación anteriormente mencionada de años y periodos en torno a la ley contra la violencia de género. Se calcularon los riesgos relativos con sus intervalos de confianza con una significación estadística del $95 \%$.

Por último, se calculó el índice epidémico mensual de muertes y denuncias de mujeres por VCI. Para ello se calculó la razón entre las muertes por VCI por meses y la mediana de muertes de los meses correspondientes en el quinquenio anterior al mes para en que se calcula el valor del índice. El índice epidémico se cálculo desde enero de 2003 hasta diciembre de 2006. Se interpretó como incidencia normal cuando el valor resultante se encontraba entre 0,76 y 1,24 ; incidencia baja si era igual o menor a 0,75 , e incidencia alta cuando el valor era igual o mayor de $1,25^{31}$. A este último se le denominó umbral epidémico. Este mismo procedimiento se llevó a cabo con los datos de denuncias. Se estimaron también los índices epidémicos subyacentes de denuncias y muertes por VCI. Para la realización de este último indicador se calculó el promedio de las puntuaciones obtenidas en los índices epidémicos de los 12 meses anteriores al mes para el que se calcula el valor del índice.

Los análisis estadísticos de los modelos de Poisson se realizaron con Egret 2.0.3. Para el resto de cálculos se utilizo Microsoft Excel 2002. 


\section{RESULTADOS}

Se observó un aumento en el número denuncias por VCI a partir del año 2002. En concreto, se pasó de 24.163 denuncias en 2001 a 43.313 en el año 2002. La tasa cruda de denuncias por VCI fue cada vez mayor, dándose las tasas más altas en los últimos dos años. Para 2005 la tasa denunciar por cada 1.000 mujeres es de 3.25 y en 2006 de 3.38 (tabla 1).

El modelo de Poisson para cada año también muestra un incremento de la posibilidad de denunciar, tomando como año de referencia el primero del estudio 1998. Se observó también una posibilidad 1,6 veces superior de denunciar en los años 2005 y 2006 que en los años comprendidos entre 1998 y 2004 (tabla 2).

Tabla 1

Frecuencias y tasas de denuncias en mujeres españolas de 15 años o más para cada año de estudio, 1998-2006

\begin{tabular}{|l|c|c|c|}
\hline & Denuncias & Tasa x 10 $^{\mathbf{6}}$ & I. C. al 95\% \\
\hline 1998 & 19.622 & 1,0664 & $1,0515-1,0813$ \\
\hline 1999 & 21.782 & 1,1838 & $1,1681-1,1995$ \\
\hline 2000 & 22.407 & 1,2178 & $1,2018-1,2337$ \\
\hline 2001 & 24.163 & 1,3132 & $1,2967-1,3298$ \\
\hline 2002 & 43.313 & 2,3540 & $2,3318-2,3761$ \\
\hline 2003 & 50.088 & 2,7222 & $2,6983-2,7460$ \\
\hline 2004 & 57.527 & 3,1265 & $3,1009-3,1520$ \\
\hline 2005 & 59.758 & 3,2477 & $3,2217-3,2738$ \\
\hline 2006 & 62.170 & 3,3788 & $3,3523-3,4054$ \\
\hline
\end{tabular}

Tabla 2

Comparación de denuncias por VCI en mujeres españolas de 15 años anual y por períodos (1998-2006)

\begin{tabular}{|l|c|c|c|}
\hline & Riesgo relativo & IC al 95\% & $\mathbf{p}$ \\
\hline 1998 & 1 & - & $<0,001$ \\
\hline 1999 & 1,0963 & $1,0753-1,1176$ & $<0,001$ \\
\hline 2000 & 1,117 & $1,0958-1,1386$ & $<0,001$ \\
\hline 2001 & 1,1848 & $1,1627-1,2073$ & $<0,001$ \\
\hline 2002 & 2,0897 & $2,0548-2,1252$ & $<0,001$ \\
\hline 2003 & 2,3721 & $2,3333-2,4116$ & $<0,001$ \\
\hline 2004 & 2,6965 & $2,6532-2,7405$ & $<0,001$ \\
\hline 2005 & 2,751 & $2,707-2,7957$ & $<0,001$ \\
\hline 2006 & 2,8286 & $2,7835-2,8743$ & $<0,001$ \\
\hline $1998-2004$ & 1 & - & $<0,001$ \\
\hline $2005-2006$ & 1,6575 & $1,6461-1,6689$ & $<0,001$ \\
\hline
\end{tabular}

A pesar de la tendencia creciente anteriormente descrita, el índice epidémico mensual de denuncias por VCI descendió a partir de enero de 2005. Incluso se registraron puntuaciones por debajo del umbral epidémico $(<1,25)$ en septiembre y diciembre de 2005 (1,24 respectivamente) y mayo $(1,23)$, junio $(1,16)$, agosto $(1,11)$, noviembre $(1,14)$ y diciembre $(1,16)$ de 2006. Las puntuaciones del índice epidémico subyacente confirman el descenso observado a partir de 2005 (figura 1).

Por su parte, las tasas anuales de muertes por cada 100.000 mujeres de 15 años o más oscilan entre el valor mínimo del año 1999 $(0,226)$ muertes por millón de mujeres, y el máximo del año 2004 (0,322) muertes (tabla 3). La tasa de muertes ajustada del periodo comprendido entre 1998 y 2004 fue de 2,99 muertes cada millón de mujeres de 15 años o más y la del segundo periodo, 2005-2006, fue de 2,97 muertes. No se observaron diferencias estadísticamente significativas en el riesgo de morir por VCI ni entre los años ni entre los periodos de estudio.

Por último, con respecto al índice epidémico mensual de muertes por VCI, se observó una tendencia decreciente a partir de enero de 2005. Sólo en los meses de noviembre de 2005 (IE=2), enero (IE=1,5), febrero $(\mathrm{IE}=1,25)$ y agosto $(\mathrm{IE}=1,29)$ de

Tabla 3

Frecuencias y tasas de mortalidad ajustadas por edad en mujeres españolas de 15 años o más para cada año de estudio

\begin{tabular}{|l|c|c|c|}
\hline & Muertes & Tasa x 10 & IC al 95\% \\
\hline 1998 & 46 & 0,26408 & $0,1878-0,3404$ \\
\hline 1999 & 40 & 0,22677 & $0,1565-0,297$ \\
\hline 2000 & 57 & 0,32009 & $0,237-0,4032$ \\
\hline 2001 & 52 & 0,28722 & $0,2092-0,3653$ \\
\hline 2002 & 47 & 0,25544 & $0,1824-0,3285$ \\
\hline 2003 & 66 & 0,35210 & $0,2672-0,4371$ \\
\hline 2004 & 61 & 0,32209 & $0,2413-0,4029$ \\
\hline 2005 & 54 & 0,28003 & $0,2053-0,3547$ \\
\hline 2006 & 61 & 0,31633 & $0,2369-0,3957$ \\
\hline
\end{tabular}


Figura 1

Índice epidémico mensual (2003-2006) ${ }^{\mathrm{a}} \mathrm{e}$ Índice Epidémico subyacente (2004-2006) ${ }^{\mathrm{b}}$ de denuncias de mujeres por Violencia del Compañero Intimo ${ }^{c}$

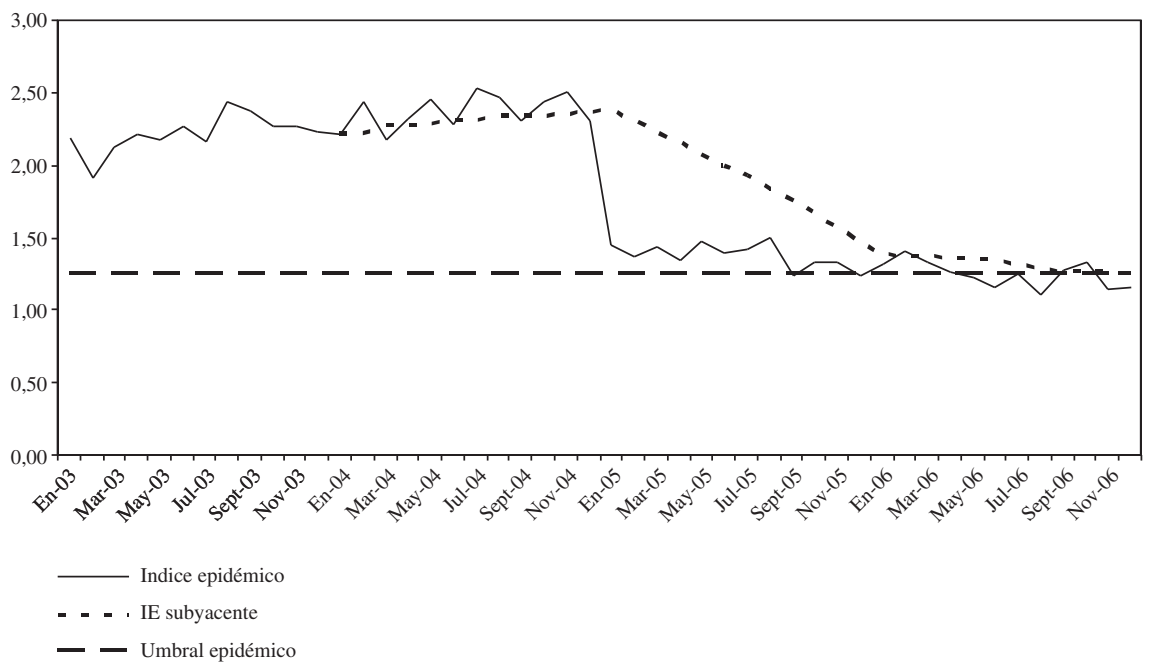

${ }^{a}$ Razón entre las muertes por VCI por meses y la mediana de muertes de los meses correspondientes en el quinquenio anterior al mes para en que se calcula el valor del índice.

${ }^{b}$ Promedio de las puntuaciones obtenidas en los índices epidémicos de los 12 meses anteriores al mes para el que se calcula el valor del índice.

${ }^{\mathrm{c}} \mathrm{Se}$ considera incidencia media cuando el valor resultante se encuentra entre 0,76 y 1,24 ; incidencia baja si es menor o igual a 0,75 e; incidencia alta si el valor es mayor o igual a 1,25 . Se identifica el umbral epidémico en 1,25 .

Figura 2

Índice epidémico mensual (2003-2006) ${ }^{\mathrm{a}} \mathrm{e}$ Índice Epidémico subyacente (2004-2006) ${ }^{\mathrm{b}}$ de muertes de mujeres por Violencia del Compañero Íntimo ${ }^{c}$

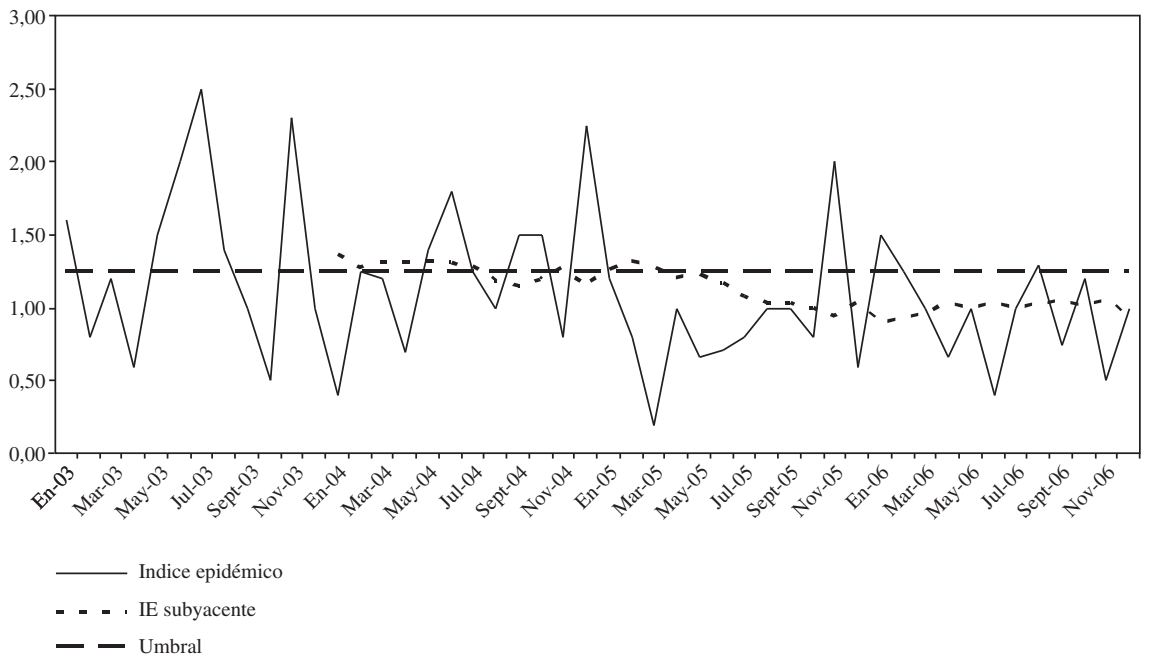

${ }^{a}$ Razón entre las muertes por VCI por meses y la mediana de muertes de los meses correspondientes en el quinquenio anterior al mes para en que se calcula el valor del índice.

${ }^{\mathrm{b}}$ Promedio de las puntuaciones obtenidas en los índices epidémicos de los 12 meses anteriores al mes para el que se calcula el valor del índice.

${ }^{\mathrm{c}} \mathrm{Se}$ considera incidencia media cuando el valor resultante se encuentra entre 0,76 y 1,24 ; incidencia baja si es menor o igual a 0,75 e; incidencia alta si el valor es mayor o igual a 1,25 . Se identifica el umbral epidémico en 1,25 . 
2006, se obtuvieron puntuaciones por encima del umbral epidémico. El índice epidémico subyacente con una figura más suavizada permite confirmar una tendencia en sus puntuaciones por debajo del umbral epidémico en los meses comprendidos entre enero de 2005 y diciembre de 2006 (figura 2).

\section{DISCUSIÓN}

En los últimos años, en España se ha incrementado la consideración de la VCI como delito que se ha de denunciar. La probabilidad de que las mujeres afectadas denuncien a sus parejas se ha incrementado en apenas diez años, siendo mayor la posibilidad de denunciar en los años posteriores a la ley contra la violencia de género de finales de 2004. Aunque también es a partir de entonces cuando se produce un descenso importante en la tendencia de las puntuaciones de los índices epidémicos mensual y subyacente de mortalidad por VCI, el riesgo de morir por esta causa no presenta cambios estadísticamente significativos. La Ley española parece haber impactado en el crecimiento epidémico de las muertes pero su efecto no ha sido suficiente como para reducir el riesgo de muerte.

La interpretación de los resultados puede verse condicionada por algunas limitaciones atribuidas a los datos de las denuncias por VCI. Se sabe que todavía son muchas las mujeres que no denuncian a sus maltratadores y, por tanto, la utilización de estos datos como aproximación a la morbilidad consecuente de la VCI podría estar minusvalorando la magnitud del problema. Además, cabe tener en cuenta las limitaciones de la fuente de información de estos datos de denuncias, que limitan la posibilidad de calcular indicadores más precisos como las tasas ajustadas por edad.

Otra limitación que puede condicionar los resultados relacionados con la distribu- ción temporal de las denuncias por VCI se relaciona con el cálculo del índice epidémico mensual. A pesar del incremento observado año a año y en el periodo 2005-2006 en la posibilidad de denunciar, se registra un descenso en las puntuaciones de los índices epidémicos mensual y subyacente desde enero de 2005. Este último resultado se relaciona con el denominador del índice epidémico. Al calcular la mediana de los casos para el denominador del índice epidémico mensual de 2005, se tuvo en cuenta tres años con un elevado volumen de denuncias (2002, 2003 y 2004) y sólo dos años con un número de casos moderado (2000 y 2001). Anteriormente, por ejemplo en el índice epidémico mensual del año 2004, fueron más los años con un moderado número de denuncias (1999, 2000 y 2001) que los años con un elevado número de denuncias (2002 y 2003). Estas diferencias condicionan el tamaño del denominador del índice epidémico mensual, siendo más grande en el caso de 2005 que en el caso de 2004.

A pesar de estas limitaciones, la aproximación realizada con los datos de las denuncias permite, en principio, concluir el éxito de las medidas desarrolladas en España para la construcción social de la violencia de género como un delito que se ha de denunciar. Esta conclusión coincide con los resultados obtenidos en un estudio realizado en torno al primer y segundo plan español contra la violencia de género (1998-2004), en el que también se observó un incremento de la posibilidad de denunciar VCI a lo largo del tiempo ${ }^{27}$. Podría decirse, por tanto, que la tendencia creciente observada en relación con las denuncias no sólo puede atribuirse a las medidas de reforzamiento de las penas a los agresores y de protección a las mujeres afectadas planteadas en la Ley de Protección Integral contra la violencia de género, sino a la política desarrollada en los últimos diez años en España en torno a la violencia de género. 
En este estudio también se han obtenido resultados similares a los observados previamente a la ley contra la violencia de género con respecto a la distribución temporal de las muertes por VCI, es decir, el riesgo de morir por esta causa se ha mantenido a lo largo del tiempo 27,28 . En un contexto internacional en el que ya se cuentan con datos que respaldan la eficacia de medidas similares a las contempladas en la ley española contra la violencia de género ${ }^{17-21}$, el descenso observado en las puntuaciones del índice epidémico de mortalidad por VCI podría tener una interpretación positiva. Sin embargo, de momento y con los datos analizados, sólo puede decirse que tras la ley española contra la violencia de género la mortalidad por esta causa se mantiene.

La tendencia observada en el riesgo de morir por esta causa, en principio, podría llevar a la conclusión de que las medidas de prevención primaria desarrolladas en España contienen ciertas limitaciones en cuanto a la reducción del riesgo de morir. Sin embargo, esta cuestión también depende de la calidad de la evidencia empírica existente en torno a la etiología de este problema, que es escasa y limita$\mathrm{da}^{32,33}$. El desarrollo de medidas preventivas eficaces requiere, por tanto, de más y mayor calidad en la información científica en torno a los factores de riesgo en torno a la conducta de los hombres que maltratan a sus parejas.

Esta primera aproximación a los efectos de la ley española contra la violencia de género en la magnitud del problema amerita un seguimiento más a largo plazo y del desarrollo de indicadores que no sólo sirvan para la vigilancia epidemiológica del problema, sino también, tal y como se ha realizado en otros países ${ }^{34}$, de la monitorización del proceso de implementación de las medidas que se proponen.

\section{AGRADECIMIENTOS}

Este trabajo ha sido posible gracias al patrocinio del Observatorio de Salud de las Mujeres del Ministerio de Sanidad y Consumo.

\section{BIBLIOGRAFÍA}

1. Krug EG, Dahlberg LL, Mercy JA, Zwi AB, Lozano R, editors. World report on violence and health. Geneva: World Health Organization; 2002. p. 5

2. Word Health Organization. Multi-country study on women's health and domestic violence against women: inicial results on prevalence, health outcomes and women's responses 2005. [accedido 3 mayo 2007] Disponible en: http://www.who.int/gender/violence/who_multicountry_study/en/index.html

3. Wingood GM, DiClemente RJ, Raj A. Adverse consequences of intimate partner abuse among women in non-urban domestic violence shelters. Am J Prev Med. 2000;19:270-5.

4. Romito P, Molzan TJ, De Marchi M. The impact of current and past interpersonal violence on women's mental health. Soc Sci Med. 2005; 60:1717-27.

5. National Center for Injury Prevention and Control. Cost of Intimate Partner Violence Against Women in the United States. Atlanta (GA): Centers for Disease Control and Prevention; 2003 [accedido 3 mayo 2007] Disponible en: http://www.cdc.gov/ncipc/pub-res/ipv_cost/IPVBook-Final-Feb18.pdf

6. Walby S. The cost of domestic violence. London: Department of Trade and Industry; 2004. En Queen Mary's School of Medicine and Dentistry. Interventions to reduce violence and promote the physical and psychosocial well-being of women who experience partner violence: a systematic review of controlled evaluations. London: Centre for Health Sciences Barts and The London; 2005 [accedido 4 mayo 2007] Disponible en: http://www.dh.gov.uk/assetRoot/04/12/74/26/04127426.pdf

7. Roberts AR, editor. Handbook of Domestic Violence Intervention Strategies. Policies, programs, and Legal Remedies. New York: Oxford University Press; 2002.

8. Instituto de la Mujer: Plan de Acción contra la violencia doméstica 1998-2000. Madrid: EI Instituto; 1999. 
9. Ministerio de Trabajo y Asuntos Sociales: II Plan Integral contra la violencia doméstica 2001-2004. Revista del Ministerio de Trabajo y Asuntos Sociales. 2001; 40: 125-34.

10. Boletín Oficial del estado. Ley Orgánica de medidas de Protección Integral contra la Violencia de Género. BOE núm 313 de 29/12/2004.

11. Duran M. El proyecto de ley orgánica de medidas de protección integral contra la violencia de género. Fundamentación Jurídico Feminista [accedido 3 julio 2007] Disponible en: http://www.cepc.es/include_mav/getfile.asp?IdFileImage $=739$

12. Smith A. It's my decision, isnt it? Violence Against Women. 2000; 6: 1384-402.

13. Durán M. La ley contra la violencia de género en el contexto internacional. Temas para el Debate. 2005; 133: 23-26.

14. Domestic Violence Laws of the World. Página web con acceso a las leyes de Argentina, Australia, Bélgica, Belize, Bermuda, Bolivia, Brasil, Brunei, Islas Cayman, Chile, China, Hong Kong, Colombia, Commonwealth, Costa Rica, República Dominicana, Ecuador, El Salvador, Alemania, Guatemala, Honduras, Irlanda, Japon, Luxemburgo, Madasgascar, Malasia, Mauritania, Méjico, Nueva Zelanda, Nicaragua, Panamá, Paraguay, Perú, Portugal, República de Corea, Rumania, Santa Lucía, Singapur, Sudáfrica, España, Trinidad y Tobago, Gran Bretaña, Uruguay y Venezuela. 2004 [accedido 10 julio 2004] Disponible en: http://annualreview.law.harvard.edu/population/d omesticviolence/domesticviolence.htm

15. Wathen CN, MacMillan HL. Intervention for Violence Against Women: Scientific Review. JAMA. 2003; 289(5): 589-600.

16. Morrisson A, Ellsberg M, Bott S. Addressing GenderBased Violence: A critical review of interventions. The World Bank Research Observer; 2007 [accedido 3 julio 2007] Disponible en: http://wbro.oxfordjournals.org/cgi/reprint/lkm003v1

17. Vittes KA, Sorenson SB. Are temporary restraining orders more likely to be issued when applications mention firearms? Eval Rev. 2006; 30(3): 266-82.

18. Vigdor ER, Mercy JA. Do laws restricting access to firearms by domestic violence offenders prevent intimate partner homicide? Eval Rev. 2006; 30(3): 313-46.

19. McFarlane J, Malecha A, Gist J, Watson K, Batten E, Hall I, Smith S. Protection orders and intimate partner violence: an 18 month study of 150 black, hispanic and white women. Am J Public Health. 2004; 94(4): 613-8.

20. Logan TK, Shannon L, Cole J, Walker R. The impact of differential patterns of physical violence and stalking on mental health and help-seeking among women with protective orders. Violence Against Women. 2006; 12(9): 866-86.

21. Kingsnorth R. Intimate partner violence: predictors of recidivism in a sample of arrestees. Violence Against Women. 2006; 12(10): 917-35.

22. Ruiz-Perez I, Mata-Pariente N, Plazaola-Castaño J. Women's Response to intimate partner violence. J Interpers Violence. 2006; 21 (9): 1156-68.

23. Ruiz-Perez I, Plazaola-Castaño J, del Rio Lozano, M. How do women in Spain deal with an abusive relationship? J Epidemiol Community Health. 2006; 60(8): 706-11

24. Balance Resultados de la Aplicación de la Ley Orgánica 1/2004, de 28 de diciembre, de medidas de protección integral contra la violencia de género. Avance. 2004 [accedido 10 julio 2007] Disponible en: http://www.mtas.es/igualdad/violencia/Balance.pdf

25. Federación de Mujeres Separadas y Divorciadas. Buscador de documentos sobre Violencia contra las Mujeres [accedido 21 abr 2007]. Disponible en: http://www.separadasydivorciadas.org/

26. Instituto de la mujer. Estadísticas sobre muertes de mujeres causadas por VCI. Informe Estadístico. [accedido 21 mayo 2007] Disponible en: http://www.mtas.es/mujer

27. Vives-Cases C, Alvarez-Dardet C, Caballero P. Violencia del Compañero Íntimo en España. Gac Sanit. 2003; 17: 268-74.

28. Vives-Cases C, Caballero P, Alvarez-Dardet C. Análisis Temporal de la Violencia del Compañero Íntimo en España. Gac Sanit. 2004; 18: 346-50.

29. INE Padrón Municipal de 1999 a 2006. Informe Estadístico. [accedido 21 abr 2007] Disponible en: http://www.ine.es

30. Hennekens C, Buring J. Epidemiology in Medicine. Boston: Little, Brown; 1987.

31. García A, Ramos J, Sánchez J, Herrera D, Martínez F. Evolución de la Vigilancia Epidemiológica de la brucelosis en Extremadura durante el año 2000. Madrid: Bol Epidemiol Sem. 2002; 10: 261 . 
32. Feldman CM. Childhood precursors of adult interpartner violence. Clin. Psychol. 1997; 4: 307-334.

33. Edleson JL. Judging the success of treatments with men who batter. In Besharov DJ, ed. Family violence: Research and public policy issues. New York, NY: AEI Press; 1990, pp. 130-145.
34. Sistemas de vigilancia de la violencia doméstica en Centroamérica. $19^{\text {a }}$ sesión del subcomité sobre la mujer, la salud y el desarrollo del comité ejecutivo. Organización Panamericana de la Salud/ Organización Mundial de la Salud. Washington, 12 al 14 marzo 2001 [accedido 30 mayo 2007] Disponible en: http://www.paho.org/spanish/gov/ce/msd/msd19_8-s.pdf 